\title{
Características cinemáticas da pedalada em ciclistas competitivos de diferentes modalidades
}

\author{
Felipe Carpes ${ }^{1}$ \\ Frederico Dagnese ${ }^{1}$ \\ Rodrigo Bini $^{2}$ \\ Fernando Diefenthaeler ${ }^{2}$ \\ Mateus Rossato ${ }^{1}$ \\ Carlos B. Mota ${ }^{2}$
}

Antônio C. S. Guimarães²

\author{
${ }^{1}$ Universidade Federal de Santa Maria \\ Laboratório de Biomecânica \\ Brasil \\ ${ }^{2}$ Universidade Federal do Rio Grande do Sul, \\ Laboratório de Pesquisa do Exercício \\ Porto Alegre \\ Brasil
}

RESUMO

O objetivo deste estudo foi comparar a cinemática de pedalada em ciclistas de duas modalidades diferentes (estrada e mountain-bike). A aquisição dos dados foi feita usando o sistema Peak Motus, sendo analisado o comportamento angular da coxa, joelho e tornozelo enquanto os ciclistas pedalaram em intensidade relativa ao segundo limiar ventilatório. Os resultados foram analisados estatisticamente com teste $t$ de Student a cada $30^{\circ}$ do pé-de-vela, não mostrando diferenças entre as modalidades. Entretanto, a cinemática do tornozelo apresentou grande variabilidade. A característica encontrada para o tornozelo possivelmente seja um fator decisivo para diferenças na técnica de pedalada entre os ciclistas destas duas modalidades, em relação à aplicação das forças no pedal.

Palavras-chave: ciclismo de estrada, ciclismo mountain-bike, cinemática.

\section{ABSTRACT}

Pedaling Kinematics Characteristics of Competitive Cyclists of Different Disciplines

The purpose of this study was to compare the pedaling kinematics of cyclists of two different disciplines (road and mountain-bike). Data collection and analysis were accomplished using the Peak Motus System, and the angular kinematics behavior for the thigh, knee and ankle were analyzed while the cyclists pedaled in relative intensity to the second ventilatory threshold. The results were statistically compared by Student's t-test to every $30^{\circ}$ of crank, showing no statistically differences among the cycling disciplines. However, the ankle kinematics presented high variability. This characteristic found for the ankle may be a decisive factor related to the differences in the pedaling technique among cyclists of these two disciplines.

Key Words: road cycling, mountain-bike cycling, kinematics. 


\section{INTRODUÇÃO}

O ciclismo é um esporte com características biomecânicas bem conhecidas. No entanto, ainda existem muitas dúvidas em relação à técnica de pedalada e sua repercussão sobre variáveis biomecânicas do desempenho do ciclista.

Em busca de soluções para algumas destas dúvidas, diversos protocolos de avaliação biomecânica da pedalada são utilizados $(4,10,13)$. Com base na cinemetria, alguns modelos para análise do movimento em duas ou três dimensões têm sido desenvolvidos, com o propósito de explicar as características mecânicas do movimento cíclico realizado pelos membros inferiores durante a pedalada $(1,8,12)$. Tais análises se tornam, ainda, mais completas quando se têm associados à cinemetria outros métodos de medição, como a dinamometria. Com isso, respostas a diferentes estímulos são registradas e analisadas de acordo com um objetivo. No esporte de rendimento os objetivos destas análises estão comumente relacionados com a maximização do desempenho, como reportado por Diefenthaeler (6), que avaliou ciclistas de estrada alterando-se a posição do selim em $1 \mathrm{~cm}$ (horizontal e verticalmente, nas duas direções), observando que as alterações na cinemática sagital do membro inferior foram pequenas e ocorreram fundamentalmente na articulação do tornozelo. Nesta avaliação, também as forças no pedal apresentaram alterações, quando modificada a posição do selim. A interface pé-pedal é o ponto firme de contato para a propulsão no ciclismo, e a posição do tornozelo pode afetar significativamente a transmissão da força gerada pelos músculos do membro inferior para o pé-de-vela (11), podendo o movimento do tornozelo ser influenciado, também, por mudanças na carga de trabalho $(7,11)$.

Comparando-se a técnica de pedalada em ciclistas de diferentes modalidades através da aplicação de força no pedal, percebe-se que ciclistas mountain-bike (MTB) apresentam uma mecânica de pedalada mais uniforme (picos de força semelhantes) e, consequentemente, uma técnica de pedalada melhor do que outros ciclistas, quando avaliados em laboratório. Isto pode, em parte, ser explicado pela maior exposição dos ciclistas MTB a subidas, que exigem uma constante produção de força $(2,3)$. Além do referido estudo, pouco se sabe sobre as características de diferentes modalidades do ciclismo, sendo que geralmente os estudos avaliam uma modalidade e generalizam os resultados para as demais. Desse modo, este estudo buscou comparar a cinemática angular do membro inferior de ciclistas das modalidades estrada e MTB.

\section{METODOLOGIA}

Para a comparação da cinemática angular de ciclistas de diferentes modalidades foram avaliados 8 ciclistas de nível estadual (Rio Grande do Sul) e nacional (Brasil), sendo que 4 eram ciclistas de estrada e 4 eram ciclistas de MTB, todos possuindo experiência em competições (mais de 7 anos).

Devido às diferentes localidades de treinamento dos ciclistas, as avaliações foram realizadas em duas fases. Na primeira, foram avaliados os ciclistas de estrada, junto ao Laboratório de Pesquisa do Exercício da Universidade Federal do Rio Grande do Sul, onde os mesmos tiveram suas bicicletas acopladas a um ciclossimulador Cateye modelo CS1000 (Cateye Co., Osaka, Japan). Na segunda fase, as avaliações foram desenvolvidas no Laboratório de Biomecânica da Universidade Federal de Santa Maria, onde foram avaliados os ciclistas de MTB. Estes pedalaram em um cicloergômetro SRM Training Systems, modelo científico (SRM Science, Welldorf, Germany) que permite o ajuste de sua geometria, a fim de apresentar a mesma geometria da bicicleta de cada ciclista avaliado.

A carga utilizada para a coleta de dados com todos os ciclistas foi correspondente ao $2^{\circ}$ limiar ventilatório individual, determinado a partir do consumo máximo de oxigênio. Este foi determinado sempre no dia prévio à avaliação cinemática, através de um protocolo de rampa (carga de $100 \mathrm{~W}$ iniciais com incrementos de $25 \mathrm{~W}$ a cada minuto e cadência de pedalada mantida entre 70 e $100 \mathrm{rpm}$ ) até exaustão voluntária máxima.

No dia seguinte, após aquecimento prévio de 10 minutos, os ciclistas foram filmados enquanto pedalavam em cadência preferida. Para a determinação dos ângulos articulares de interesse, a coleta dos dados foi feita através de cinemetria, com uso da videografia bidimensional para a aquisição das imagens, que foi realizada com uma câmera (Peak HSC180) operando em taxa de amostragem de $180 \mathrm{~Hz}$. A 
câmera foi posicionada perpendicularmente ao plano de movimento, a uma distância de aproximadamente $4 \mathrm{~m}$, permitindo a aquisição de imagens adequadas para o cálculo das variáveis cinemáticas no plano sagital do hemicorpo direito, ao longo de 10 ciclos consecutivos de pedalada.

Para a filmagem da pedalada foram fixados marcadores reflexivos em pontos anatômicos de referência no trocânter maior, epicôndilo lateral, maléolo lateral, IV metatarso, calcâneo, centro de rotação do pedal e centro de rotação do pé-de-vela. Os marcadores reflexivos do calcâneo e IV metatarso foram fixados sobre a sapatilha dos ciclistas, imediatamente sobre o ponto anatômico de referência. Foram analisados os ângulos do quadril (ângulo absoluto da coxa em relação ao eixo horizontal), do joelho (ângulo relativo interno entre a coxa e a perna), e do tornozelo (ângulo relativo entre o segmento perna e o segmento pé), conforme ilustra a figura 1. A posição do péde-vela também foi determinada com o auxílio da cinemetria. A digitalização das imagens, através do reconhecimento automático dos marcadores reflexivos de referência, e os cálculos de todas as variáveis cinemáticas selecionadas foram feitos por meio do Sistema Peak Motus (Peak Performance Technologies, Englewood, USA).

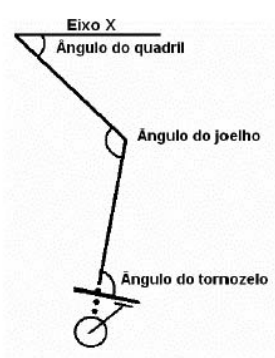

Figura 1: llustração dos ângulos articulares calculados.

As coordenadas espaciais obtidas para os pontos de referência foram submetidas à filtragem (filtro Butterworth de $4^{\mathrm{a}}$ ordem, com frequiência de corte de $6 \mathrm{~Hz}$ ) e utilizadas para o cálculo dos ângulos articulares. Posteriormente, os dados angulares foram exportados para o software Origin Professional 6.0 (Microcal Inc., USA), onde foram analisados de acordo com a posição angular do pé-de-vela e apresentados graficamente. A discussão dos resultados foi desenvolvida em função de cada quadrante do ciclo de pedalada (figura 2).

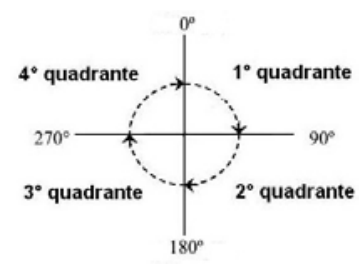

Figura 2: Quadrantes do ciclo de pedalada $\left(0^{\circ}-180^{\circ}\right.$ fase de propulsão $180^{\circ}-360^{\circ}$ fase de recuperação].
A análise estatística envolveu a comparação entre os grupos, utilizando-se o teste t de Student para amostras independentes, comparando as variáveis angulares entre as duas modalidades a cada $30^{\circ}$ do ciclo de pedalada, separadamente, por meio do pacote estatístico Statistica 5.1 (Statsoft, Inc, USA), com um nível de significância igual a 0,05 . O coeficiente de variação apresenta a razão entre o desvio-padrão e a média para o ângulo articular ao longo do ciclo de pedalada e foi utilizado para indicar a variabilidade dos dados.

\section{RESULTADOS}

Na tabela 1 estão apresentadas as variáveis descritivas do comportamento angular da coxa. Não foram observadas diferenças estatisticamente significativas entre os ciclistas de estrada e MTB avaliados.

Tabela 1: Variáveis angulares da coxa para os ciclistas de estrada e de MTB, expressos em média [desvio-padrão] e coeficiente de variação [\%] de 10 ciclos de pedalada.

\begin{tabular}{lcc} 
Ângulo da coxa & Estrada & Mountain-bike \\
\hline Flexão $\left(^{\circ}\right)$ & $17(4)$ & $23(3)$ \\
Extensão $\left(^{\circ}\right)$ & $61(2)$ & $61(3)$ \\
Amplitude de movimento $\left(^{\circ}\right)$ & $44(5)$ & $38(5)$ \\
Coeficiente de variação $(\%)$ & 10 & 9
\end{tabular}

Na figura 3 é apresentado o comportamento angular da coxa, ao longo do ciclo de pedalada, para o grupo de ciclistas de estrada e de MTB. De acordo com os resultados apresentados para o coeficiente de variação (tabela 1), percebe-se que os grupos apresentaram homogeneidade, o que se refletiu no gráfico, onde um padrão semelhante foi observado para todos os ciclistas. 


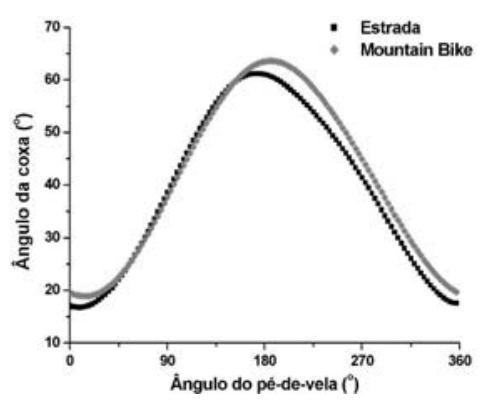

Figura 3: Comportamento angular da coxa dos ciclistas de estrada e MTB.

Ainda em relação ao comportamento angular observado para a coxa nos ciclistas avaliados, as figuras 4 e 5 ilustram os resultados individuais de cada ciclista da amostra, das modalidades estrada e MTB, respectivamente.

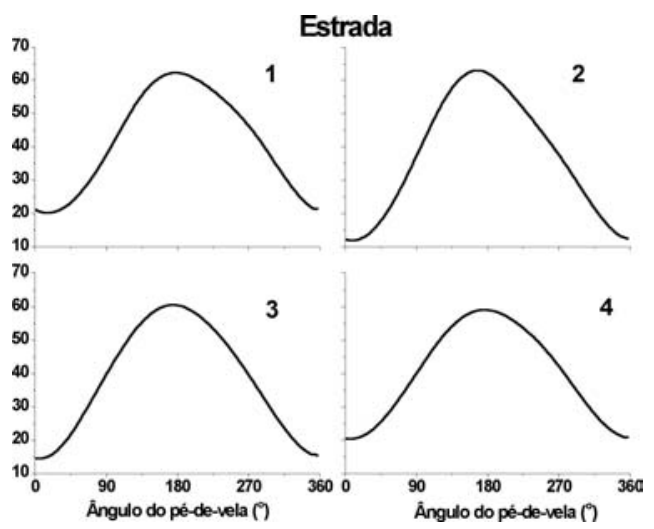

Figura 4: Comportamento angular da coxa de cada ciclista de estrada avaliado.

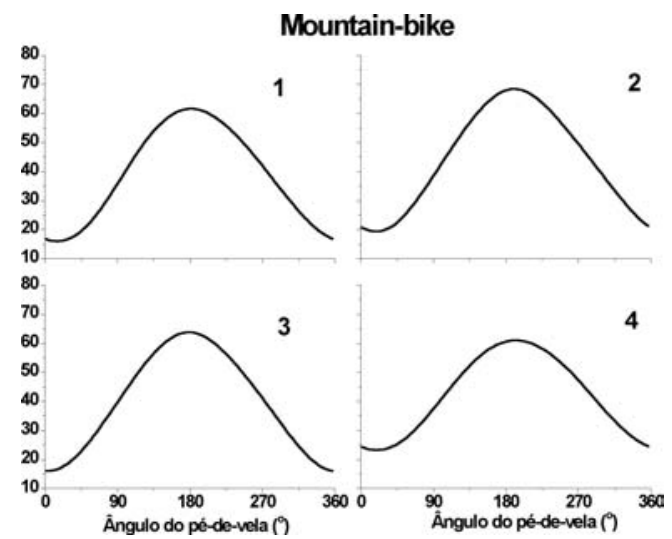

Figura 5: Comportamento angular da coxa de cada ciclista MTB avaliado.
Tanto os ciclistas de estrada quanto os de MTB apresentaram, em média, o mesmo grau de extensão da coxa, no entanto, os ciclistas de MTB apresentaram uma menor flexão, que acarretou uma menor amplitude de movimento. Para o comportamento angular do joelho, não foram encontradas diferenças entre os ciclistas de estrada e MTB. Novamente os ciclistas apresentaram resultados muito semelhantes (tabela 2).

Tabela 2: Variáveis angulares do joelho para os ciclistas de estrada e de MTB, expressos em média [desvio-padrão] e coeficiente de variação [\%] de 10 ciclos de pedalada.

\begin{tabular}{lcc} 
Ângulo do joelho & Estrada & Mountain Bike \\
\hline Mínimo $\left({ }^{\circ}\right)$ & $69(3)$ & $69(3)$ \\
Máximo $\left({ }^{\circ}\right)$ & $140(4)$ & $142(9)$ \\
Amplitude $\left(^{\circ}\right.$ ) & $70(3)$ & $72(8)$ \\
Coeficiente de variação (\%) & 3 & 5
\end{tabular}

Na figura 6 é apresentado o comportamento angular do joelho ao longo do ciclo do pé-de-vela, em média, para o grupo de estrada e de MTB. Nas figuras 7 e 8 , os padrões individuais do comportamento angular do joelho são apresentados para os ciclistas de estrada e de MTB, respectivamente.

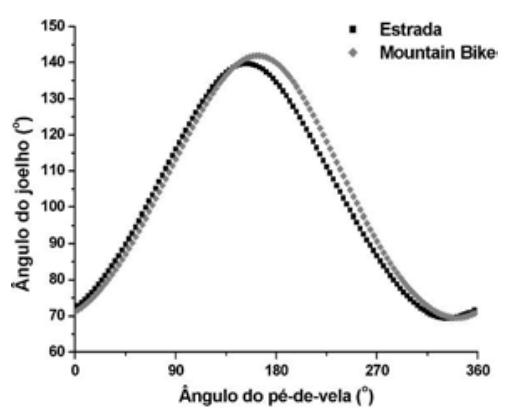

Figura 6: Comportamento angular do joelho de ciclistas de estrada e MTB. 


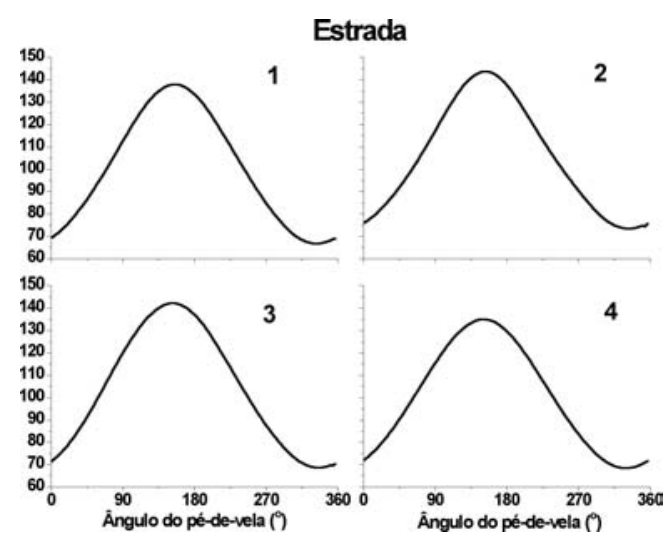

Figura 7: Comportamento angular do joelho de cada ciclista de estrada avaliado.

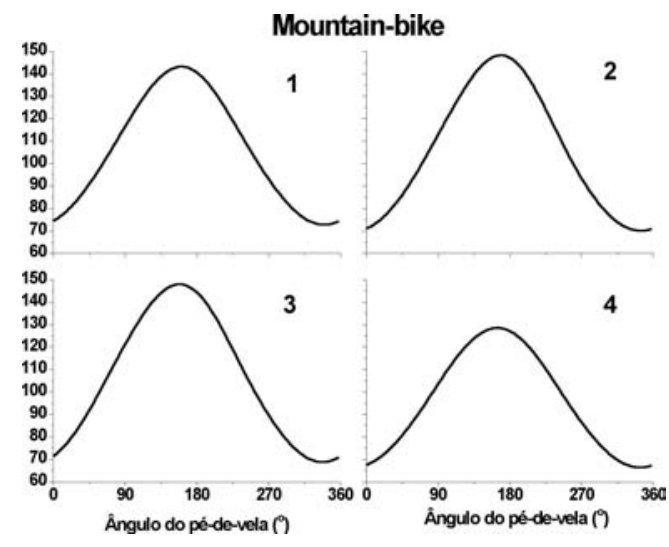

Figura 8: Comportamento angular do joelho de cada ciclista MTB avaliado.

O padrão angular do joelho foi similar ao da coxa. A máxima extensão, em ambas as modalidades, ocorreu antes dos $180^{\circ}$ (a partir da metade do segundo quadrante). Para ambas as modalidades, a máxima flexão ocorreu um pouco antes dos $360^{\circ}$ (a partir da metade do quarto quadrante). Novamente uma amplitude de movimento um pouco maior foi reportada para os ciclistas MTB.

$\mathrm{Na}$ articulação do tornozelo não foram observadas diferenças estatisticamente significativas, o que pode ter ocorrido devido ao grande coeficiente de variação observado nos dois grupos. De acordo com o apresentado na tabela 3, o coeficiente de variação foi muito alto (maior que $70 \%$ ), indicando que a movimentação do tornozelo foi muito específica para cada ciclista.
Tabela 3: Variáveis angulares do tornozelo para ciclistas de estrada e de MTB, expressos em média (desvio-padrão) e coeficiente de variação [\%] de 10 ciclos de pedalada.

\begin{tabular}{lcc} 
Ângulo do tornozelo & Estrada & Mountain Bike \\
\hline Mínimo $\left(^{\circ}\right)$ & $-8(4)$ & $-3(12)$ \\
Máximo $\left(^{\circ}\right)$ & $8(18)$ & $11(9)$ \\
Amplitude $\left(^{\circ}\right)$ & $16(14)$ & $14(5)$ \\
Coeficiente de variação $(\%)$ & $7 ?$ & 76
\end{tabular}

Na figura 9 é ilustrado o comportamento angular do tornozelo, em ambos os grupos, ao longo do ciclo do pé-de-vela. A variabilidade entre os ciclistas foi tamanha que forçou a apresentação dos gráficos com escalas individuais para cada ciclista, a fim de mostrar de forma mais clara o comportamento observado (figuras 10 e 11).

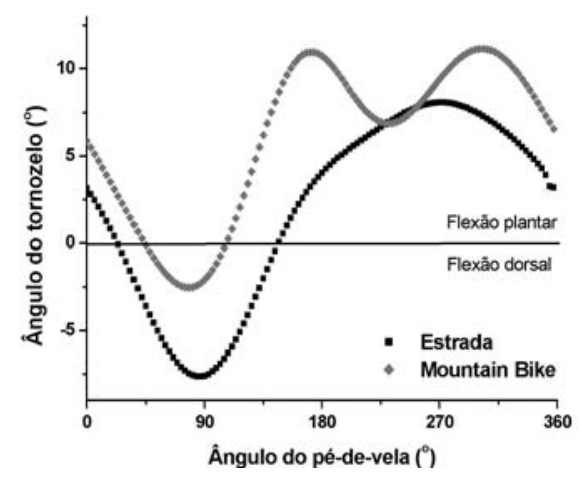

Figura 9: Comportamento angular do tornozelo de ciclistas de estrada e MTB.

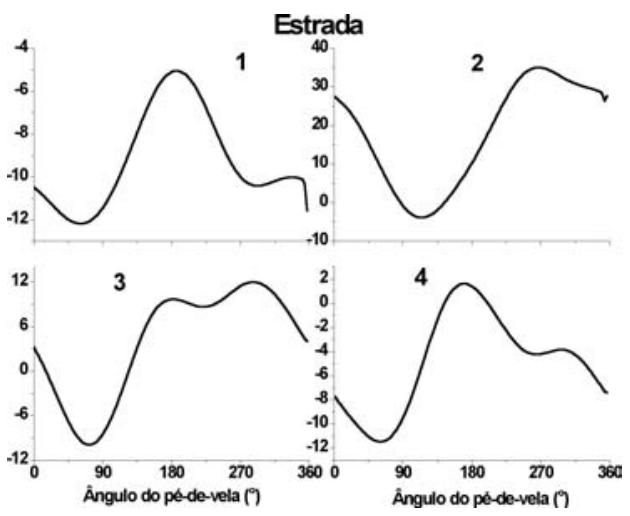

Figura 10: Comportamento angular do joelho de cada ciclista de estrada avaliado. 


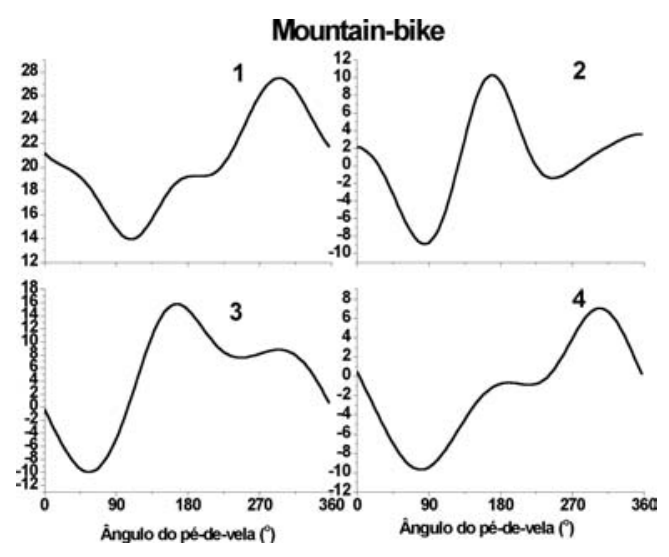

Figura 11: Comportamento angular do tornozelo de cada ciclista de MTB avaliado.

A análise da movimentação da articulação do tornozelo dos ciclistas avaliados permite verificar um aspecto interessante: foi nesta articulação que ocorreram as maiores alterações angulares ao longo do ciclo do pé-de-vela e onde as mesmas se apresentaram de modo mais proeminente, devido à grande variabilidade observada. Os ciclistas de estrada apresentaram maior flexão dorsal, enquanto que os ciclistas de MTB apresentaram maior flexão plantar. Em ambos os grupos, a maior flexão dorsal ocorreu ligeiramente antes dos $90^{\circ}$ do ciclo do pé-de-vela, sendo observado um padrão semelhante até aos $180^{\circ}$ do pé-de-vela. $\mathrm{Na}$ fase de recuperação da pedalada (de $180^{\circ}$ a $360^{\circ}$ do ciclo do pé-de-vela), algumas divergências, ainda que pequenas, foram observadas.

\section{DISCUSSÃO}

O comportamento diferenciado das variáveis avaliadas reforça a validade de uma discussão individualizada e focada em cada uma delas.

O comportamento angular da coxa foi semelhante nas duas modalidades. Logo após o início do ciclo do péde-vela, a articulação do quadril apresenta sua máxima extensão, próximo aos $180^{\circ}$ do pé-de-vela, retornando a flexão no ponto morto superior, início do ciclo. O fato da máxima extensão do quadril ter ocorrido após os $180^{\circ}$ na modalidade MTB deve-se, provavelmente, a uma regulagem do selim um pouco mais à frente ou um pouco mais baixo, o que é uma das características do posicionamento nesta modalidade. Um selim ajustado de acordo com a regulagem proposta por Burke e Pruitt (5), dificulta o posicionamento comumente utilizado por atletas de MTB quando competindo em trajetos irregulares e com descidas (onde os ciclistas tomam uma posição de suspensão do corpo sem contato com o selim, e sim ligeiramente atrás e abaixo do mesmo), visando uma melhor estabilidade na bicicleta. Esta estratégia pode fazer com que, na posição de referência (6), o posicionamento do membro inferior esteja suscetível a uma maior sobrecarga, principalmente na articulação patelo-femoral $(5,7,8)$. Na modalidade estrada, um ajuste estanque, de acordo com o proposto pela literatura (5) e aliado às características individuais, é possível, pois raramente o ciclista de estrada modifica seu posicionamento na bicicleta ao longo de uma prova. Novamente o padrão de movimento da articulação do tornozelo pode ter contribuído para que menores variações angulares fossem obtidas, quando em comparação com a modalidade de MTB.

Observa-se que o joelho dos ciclistas não apresenta extensão completa durante a pedalada, característica que está ligada à capacidade de produção de força em função do ângulo de inserção muscular e minimização da compressão patelar (5). De acordo com Pierson-Carey e colaboradores (11), durante a pedalada, a máxima flexão plantar ocorrerá no final da fase de potência $\left(180^{\circ}\right.$ do ciclo do pé-de-vela) e a máxima flexão dorsal ocorrerá no fim da fase de recuperação $\left(360^{\circ}\right.$ do ciclo do pé-de-vela). Neste estudo, isso nem sempre foi observado, entretanto, ciclistas de estrada apresentaram uma maior flexão dorsal, enquanto que ciclistas de MTB apresentaram uma maior flexão plantar durante o ciclo de pedalada. Infere-se que esta característica possa ser um dos fatores que explique as diferenças encontradas na aplicação de força nos pedais entre diferentes modalidades $(2,3)$, principalmente quando se leva em consideração que o movimento do tornozelo é uma importante condição mecânica para permitir maior aplicação de força resultante no pedal, em particular na fase de propulsão da pedalada. Indicando também que os ciclistas podem otimizar o desempenho na pedalada usando estratégias de movimentação do tornozelo (11), justamente por esta ser a articulação com maior liberdade de movimento em relação às 
outras do membro inferior durante a pedalada. Os ciclistas de MTB parecem sustentar o maior tempo possível uma posição de flexão plantar do tornozelo, buscando com isso manter a ação de "puxar" o pedal na recuperação. Já nos ciclistas de estrada, a posição do tornozelo no início do ciclo parece influenciar uma maior ação dos extensores da coxa, com o objetivo de antecipar a ação destes e empurrar o pedal para a frente, pouco antes do ponto morto superior, a fim de otimizar a força, de acordo com a posição do tornozelo. Com essa análise ficaria subentendido que os atletas encontram em seus músculos extensores um maior aproveitamento de suas características potenciais. As características angulares observadas para ambas as modalidades podem estar condicionadas a fatores externos, como a geometria do quadro da bicicleta, o qual no ciclismo MTB é menor, bem como ao trajeto em uma competição e à intensidade de esforço (9). O comportamento diferenciado entre os ciclistas, para a articulação do tornozelo, indica que mesmo atletas de nível competitivo semelhante, como os indivíduos deste estudo, possuem estratégias musculares diferenciadas, as quais podem implicar em diferenças na sua técnica de pedalada, principalmente a aplicação de força nos pedais. Uma importante observação a se fazer é que ciclistas MTB realizam grande parte de seus treinamentos utilizando bicicletas de estrada, e essa transição de uma geometria de quadro para a outra deveria ser melhor avaliada, pois pode exercer influência na técnica de pedalada.

\section{CONCLUSÕES}

Através de uma análise cinemática comparativa entre as modalidades de estrada e MTB, pode-se notar que, para as articulações do quadril e joelho, o padrão de movimento é muito semelhante entre as duas modalidades avaliadas, apresentando também pequena variabilidade entre os ciclistas avaliados. Por outro lado, a articulação do tornozelo, ainda que o comportamento angular não tenha diferido estatisticamente entre as modalidades, apresentou peculiaridades para cada modalidade e, também, uma grande variabilidade entre os ciclistas, podendo o padrão de movimentação do tornozelo ser um fator determinante das diferenças na técnica de pedalada nas modalidades avaliadas.
Com isso, infere-se que diferentes estratégias musculares podem ser observadas entre as modalidades, bem como entre ciclistas de uma mesma modalidade, sugerindo que este tipo de comparação seja feito considerando a atividade muscular durante a pedala$\mathrm{da}$, a fim de prover mais informações para explicar as diferenças na técnica de pedalada observadas entre as modalidades e permitir o uso de treinamentos específicos.

\section{AGRADECIMENTOS}

Gostaríamos de dedicar este trabalho ao grande mestre, Professor Antônio Carlos Stringhini Guimarães, por todos os ensinamentos e exemplos deixados. O Professor Guimarães faleceu nos dias prévios à submissão deste artigo, deixando saudade, lembrança e principalmente orgulho de tê-lo tido como professor, colega e, acima de tudo, amigo.

\section{CORRESPONDÊNCIA}

\section{Felipe Pivetta Carpes}

Universidade Federal de Santa Maria

Centro de Educação Física e Desportos

Laboratório de Biomecânica, Prédio 51, sala 1007

Faixa de Camobi, km 9

97105-900, Santa Maria - RS

BRASIL

felipecarpes@gmail.com 


\section{REFERÊNCIAS}

1. Bailey MP, Maillardet FJ, Messenger N (2003). Kinematics of cycling in relation to anterior knee pain and patellar tendonitis. J Sports Sci 12:649-657.

2. Broker JP (2003) Cycling Biomechanics: Road and Mountain. In Burke ER (Ed.) High Tech Cycling. Champaign, Il.: Humans Kinetics, 119-146.

3. Broker JP, Crawley JD, Coughlin KD (2002) Pedaling mechanics differences across cycling disciplines: observations over 10 years of testing. Med Sci Sports Exerc 34(5), supplement 1, S90.

4. Broker JP, Gregor RJ (1996) Cycling Biomechanics. In Burke ER (Ed.) High Tech Cycling. Champaign, Il.: Human Kinetics, 145-165.

5. Burke ER, Pruitt AL (2003) Body positioning for cycling. In Burke ER (Ed.) High Tech Cycling. Champaign, Il.: Humans Kinetics, 69-92.

6. Diefenthaeler F (2004) Avaliação dos efeitos da posição do selim na técnica de pedalada de ciclistas: estudo de casos. Dissertação de Mestrado, Universidade Federal do Rio Grande do Sul, Porto Alegre, RS, Brasil.

7. Ericson MO, Nisell R, Nemeth G (1988) Joint motions of the lower limb during ergometer cycling. J Orthop Sports Phys Therapy 9:273-278.
8. Gregersen CS, Hull ML (2003) Non-driving intersegmental knee moments in cycling computed using a model that includes three-dimensional kinematics of the shank/foot and the effect of simplifying assumptions. J Biomech 36:803-813.

9. Impellizzeri F, Sassi A, Rodriguez-Alonso M, Mognoni P, Marcora S (2002) Exercise intensity during off-road cycling competitions. Med Sci Sports Exerc 34(11):18081813.

10. Lafortune MA, Cavanagh PR (1983). Effectiveness and efficiency during bicycle riding. In Matsui \& Kobashi K (Ed.) Biomechanics VIII-B. Champaign, Il: Human Kinetics, 928-936.

11. Pierson-Carey CD, Brown DA, Dairaghi CA (1997). Changes in resultant pedal reaction forces due to ankle immobilization during pedaling. J App Biomech 13(3):334346.

12. Ruby P, Hull ML, Hawkins D (1992). Three dimensional knee loading during seated cycling. J Biomech 25:41-53.

13. Sanderson DJ (1991). The influence of cadence and power output on the biomechanics of force application during steady-state cycling in competitive and recreational cyclists. J Sport Sci 9: 191-203. 